\title{
PRODUÇÃO DE PAINÉIS DE MADEIRA AGLOMERADA DE Grevillea robusta A. Cunn. ex R. Br. ${ }^{1}$
}

\author{
Setsuo Iwakiri² ${ }^{2}$ Jarbas Shimizu ${ }^{3}$, José de Castro Silva ${ }^{4}$, Cláudio Henrique Soares Del Menezzi ${ }^{4}$, Carlos \\ Augusto Puehringher ${ }^{5}$, Ivan Venson ${ }^{5}$ e Christine Larroca ${ }^{6}$
}

\begin{abstract}
RESUMO - Este trabalho teve como objetivo avaliar o comportamento da madeira de Grevillea robusta na produção de painéis de madeira aglomerada. Os painéis foram produzidos em densidades de 0,60 e $0,80 \mathrm{~g} / \mathrm{cm}^{3}$ e conteúdo de resina de 6 e $8 \%$. Os resultados de propriedades físicas e mecânicas dos painéis fabricados com densidade de $0,80 \mathrm{~g} / \mathrm{cm}^{3}$ e conteúdo de resina de $8 \%$ evidenciaram que a madeira de Grevillea robusta pode ser utilizada como fonte alternativa de matéria-prima para produção de painéis aglomerados.
\end{abstract}

Palavras-chave: Grevillea, painéis de madeira aglomerada e conteúdo de resina.

\section{PARTICLEBOARD MANUFACTURING FROM Grevillea robusta A. Cunn. ex R. Br.}

\begin{abstract}
The objective of this study was to evaluate the feasibility of the use of Grevillea robusta for particleboard manufacturing. The boards were manufactured in the densities of 0,60 and $0,80 \mathrm{~g} / \mathrm{cm}^{3}$ and resin contents of 6 and $8 \%$. The results of physical and mechanical properties of the boards manufactured with $0,80 \mathrm{~g} / \mathrm{cm}^{3}$ density and $6 \%$ resin content, showed that Grevillea robusta wood may be used as alternative raw material for particleboard manufacturing.
\end{abstract}

Key words: Grevillea, particleboard, resin content.

\section{INTRODUÇÃO}

Os estudos concernentes ao potencial tecnológico de espécies alternativas de rápido crescimento para usos múltiplos são de grande importância, tendo em vista aumentar a oferta de matéria-prima ao setor de indústrias de base florestal, além de contribuir para minimizar as pressões ambientais sobre o uso de florestas nativas.

Os Estados do Paraná e Santa Catarina possuem a maior área de florestas plantadas de pinus do Brasil, no entanto a demanda pela madeira também é significativa e crescente, em face do grande número de indústrias instaladas na região que a utilizam para produção de grande variedade de produtos, como: papel e celulose, lâminas, compensados, madeiras serradas e beneficiadas e painéis aglomerados e de fibras (MDF).

As indústrias de painéis aglomerados e de fibras, no Brasil, consomem um volume significativo de madeiras provenientes de florestas plantadas de pinus e algumas espécies de eucalipto. Para atender à demanda cada vez mais crescente pela madeira, há a necessidade de

\footnotetext{
${ }^{1}$ Recebido em 14.12.2002 e aceito para publicação em 10.08.2004.

2 Departamento de Engenharia e Tecnologia Florestal da Universidade Federal do Paraná - UFPR. E-mail: <setsuo@ floresta.ufpr.br>.

${ }^{3}$ Embrapa Florestas.

${ }^{4}$ Programa de Pós-Graduação em Ciências Florestais da UFPR.

${ }^{5}$ Programa de Pós-Graduação em Ciências Florestais da UFPR.

${ }^{6}$ Av. Lothário Meissner, 3400, J. Botânico - 80210-170 Curitiba, PR.
} 
não somente aumentar a área de plantios com essas espécies, mas também procurar opções de outras espécies de rápido crescimento que possam contribuir, de formas quantitativa e qualitativa, para suprir as necessidades das indústrias.

A espécie é uma das variáveis mais importantes no processo de produção de aglomerados. A relação entre a densidade do painel e a densidade da madeira utilizada é denominada de razão de compactação e indica o grau de densificação das partículas de madeira na estrutura do painel e afetará as propriedades e qualidades desta (MALONEY, 1993; MOSLEMI, 1974; TSOUMIS, 1991). Ainda, segundo esses autores, a razão de compactação deve estar na faixa de 1,3 a 1,6 para que ocorram adequada densificação e consolidação do painel na espessura final desejada. De acordo com Kelly (1977), as propriedades mecânicas dos painéis apresentam relação direta com a razão de compactação, mas as propriedades físicas exibem uma relação inversamente proporcional.

Outro efeito da espécie é em relação à área superficial específica das partículas de madeira. Considerandose painéis com as mesmas dimensões e densidade, o uso da espécie mais densa resultará na redução do volume de partículas e, conseqüentemente, da sua área superficial específica. Nessas condições, a aplicação do mesmo conteúdo de resina aumenta a sua disponibilidade por unidade de área superficial das partículas, podendo resultar em painéis com melhores propriedades, compensando o efeito da redução na razão de compactação (MOSLEMI, 1974; MALONEY, 1993).

Moslemi (1974) apresentou uma equação matemática para o cálculo da densidade do material (madeira) para mistura de espécies de diferentes densidades. Com base nessa equação, torna-se possível a mistura de espécies de maior e menor densidade para produção de painéis com razão de compactação dentro da faixa considerada adequada. Na pesquisa realizada com a mistura de madeiras de Pinus elliottii Engelm. e Mimosa scabrella Benth., Iwakiri et.al. (1995) concluíram que a mistura dessas espécies, em proporção de 50\% cada, resulta em painéis com propriedades físicomecânicas satisfatórias dentro dos padrões requeridos para utilização.

Esta pesquisa foi desenvolvida com o objetivo de avaliar o comportamento da madeira de Grevillea robusta para produção de painéis de madeira aglomerada com diferentes densidades e conteúdos de resina.

\section{MATERIAL E MÉTODOS}

\subsection{Material}

A madeira de Grevillea robusta, com idade de 6 anos, foi obtida do plantio experimental da EMBRAPA FLORESTAL, localizado no município de Foz do Iguaçu,PR. Para a manufatura dos painéis, utilizouse a resina uréia-formaldeído, com viscosidade de 560 cp, teor de sólidos de $66 \%$ e pH de 7,4.

\subsection{Manufatura dos painéis de madeira aglomerada}

As partículas de madeira foram geradas com as seguintes dimensões nominais: comprimento de $25 \mathrm{~mm}$, espessura de $0,7 \mathrm{~mm}$ e largura variável. Após a secagem ao teor de umidade aproximado de $3 \%$, as partículas foram reduzidas no moinho de martelo e, posteriormente, peneiradas para a remoção de "finos".

Os painéis foram produzidos com as seguintes dimensões: comprimento de $510 \mathrm{~mm}$, largura de 390 mm e espessura de $15 \mathrm{~mm}$. As quantidades de materiais foram determinadas para densidades dos painéis de 0,60 e $0,80 \mathrm{~g} / \mathrm{cm}^{3}$ e com conteúdo de resina sólida de 6 e $8 \%$ base peso seco das partículas de madeira, conforme demonstrado no Quadro 1. Os parâmetros do ciclo de prensagem foram estabelecidos como se segue: temperatura $=140^{\circ} \mathrm{C}$, pressão específica $=40$ $\mathrm{kgf} / \mathrm{cm}^{2}$ e tempo de prensagem $=8$ minutos.

\subsection{Ensaios físico-mecânicos dos painéis}

Após a prensagem, os painéis foram acondicionados em câmara climática até atingir o teor de umidade de equilíbrio de $12 \%$. Em seguida, foram retirados os corpos de prova para a realização de seguintes ensaios físico-mecânicos: absorção de água e inchamento em espessura após 2 e 24 horas de imersão em água, ligação interna e flexão estática. As dimensões dos corpos de prova e os procedimentos de ensaios foram estabelecidos de acordo com a norma ASTM D-1037 (1982). A análise estatística foi realizada de acordo com o delineamento inteiramente casualizado, e os resultados foram avaliados através da análise de variância e teste de Tukey a $95 \%$ de probabilidade. 
Quadro 1 - Plano experimental Table 1 - Experimental chart

\begin{tabular}{cccc}
\hline Tratamento & $\begin{array}{c}\text { Densidade } \\
\left(\mathrm{g} / \mathrm{cm}^{3}\right)\end{array}$ & $\begin{array}{c}\text { Resina } \\
(\%)\end{array}$ & $\begin{array}{c}\mathrm{N}^{\mathbf{0}} \\
\text { painéis }\end{array}$ \\
\hline 1 & 0,60 & 6 & 3 \\
2 & 0,60 & 8 & 3 \\
3 & 0,80 & 6 & 3 \\
4 & 0,80 & 8 & 3 \\
\hline
\end{tabular}

\section{RESULTADOS E DISCUSSÕES}

\subsection{Densidades da madeira e dos painéis}

Os valores médios da densidade básica da madeira e densidade aparente dos painéis aglomerados produzidos com as densidades nominais de $0,60 \mathrm{~g} / \mathrm{cm}^{3}$ e $0,80 \mathrm{~g} / \mathrm{cm}^{3}$ estão apresentados no Quadro 2 .

A densidade básica média de $0,52 \mathrm{~g} / \mathrm{cm}^{3}$ obtida para amostras de madeira utilizada nesta pesquisa pode ser considerada baixa para a espécie de Grevillea. Entretanto, deve ser considerada que as amostras foram obtidas de árvores com apenas 6 anos de idade.

Com relação à densidade média dos painéis, podese constatar que os valores são um pouco inferiores em comparação com a densidade nominal estabelecida no plano experimental. Essa diferença pode ser atribuída, principalmente, à perda de partículas durante o manuseio, nas fases anteriores à consolidação do painel na prensa quente, em razão das condições laboratoriais de manufatura.

\subsection{Propriedades físicas dos painéis}

Os valores médios de absorção de água e inchamento em espessura após 2 e 24 horas de imersão em água estão apresentados no Quadro 3.

Com base nos resultados do Quadro 3, pode-se
Quadro 2 - Valores médios das densidades da madeira e dos painéis

Table 2 - Average values of wood density and of the boards

\begin{tabular}{lc}
\hline Densidade & Valor Médio $\left(\mathrm{g} / \mathrm{cm}^{3}\right)$ \\
\hline Madeira & 0,52 \\
Painel (T1 e T2) & 0,58 \\
Painel (T3 e T4) & 0,77 \\
\hline
\end{tabular}

$\mathrm{T} 1$ e $\mathrm{T} 2=$ densidade nominal de $0,60 \mathrm{~g} / \mathrm{cm}^{3}$ e $\mathrm{T} 3$ e $\mathrm{T} 4=$ densidade nominal de $0,80 \mathrm{~g} / \mathrm{cm}^{3}$.

constatar que os painéis produzidos com densidade de $0,58 \mathrm{~g} / \mathrm{cm}^{3}$ apresentaram menor absorção de água em relação aos painéis com densidade de $0,77 \mathrm{~g} / \mathrm{cm}^{3}$, nos ensaios com duas horas de imersão em água. A princípio, essas diferenças podem estar relacionadas ao maior volume de madeira contido nos painéis com densidade de $0,80 \mathrm{~g} / \mathrm{cm}^{3}$, aumentando a área de contato superficial com a água. No entanto, após o relaxamento da estrutura densificada do painel devido ao aumento no tempo de exposição à água de 2 para 24 horas não foram constatadas diferenças estatisticamente significativas entre os painéis produzidos com densidade de 0,60 e $0,80 \mathrm{~g} / \mathrm{cm}^{3}$. Não foram constatadas diferenças estatisticamente significativas em absorção de água para painéis produzidos com diferentes conteúdos de resina.

Com relação ao inchamento em espessura, não foram constatadas diferenças estatisticamente significativas entre os valores médios de inchamento em espessura, tanto para duas horas quanto para 24 horas de imersão em água. Os fatores densidade e conteúdo de resina não interferiram estatisticamente nos resultados dessa propriedade. Entretanto, cabe ressaltar que, em termos de médias absolutas, os painéis produzidos com maior conteúdo de resina apresentaram tendências para menor inchamento em espessura.

Quadro 3 - Valores médios das propriedades físicas dos painéis Table 3 - Average values of board physical properties

\begin{tabular}{lccc}
\hline Tratamento & AA $-2 \mathrm{~h}(\%)$ & AA $-24 \mathrm{~h}(\%)$ & IE $-2 \mathrm{~h}(\%)$ \\
$\mathrm{T} 1(\mathrm{D}=0,6 / \mathrm{R}=6)$ & $15,32 \mathrm{a}$ & $51,59 \mathrm{a}$ & $10,30 \mathrm{ab}$ \\
$\mathrm{T} 2(\mathrm{D}=0,6 / \mathrm{R}=8)$ & $15,81 \mathrm{a}$ & $42,91 \mathrm{a}$ & $8,57 \mathrm{a}$ \\
$\mathrm{T} 3(\mathrm{D}=0,8 / \mathrm{R}=6)$ & $24,03 \mathrm{~b}$ & $51,65 \mathrm{a}$ & $1 \%)$ \\
$\mathrm{T} 4(\mathrm{D}=0,8 / \mathrm{R}=8)$ & $27,70 \mathrm{~b}$ & $66,59 \mathrm{a}$ & $15,32 \mathrm{~b}$ \\
\hline
\end{tabular}

Médias seguidas pela mesma letra não diferem estatisticamente entre si, a $95 \%$ de probabilidade.

$\mathrm{D}=$ densidade nominal do painel $\left(\mathrm{g} / \mathrm{cm}^{3}\right)$ e $\mathrm{R}=$ conteúdo de resina $(\%)$.

$\mathrm{AA}=$ absorção de água e IE = inchamento em espessura. 
Os resultados de inchamento em espessura ( $24 \mathrm{~h}$ ) obtidos nesta pesquisa foram inferiores em comparação com os apresentados por Iwakiri et al. (1999) e (2000), para painéis aglomerados de pinus e de eucalipto com densidade de $0,70 \mathrm{~g} / \mathrm{cm}^{3}$, cujos valores médios foram: pinus $=32,24 \%$ e eucalipto $=34,95 \%$.

\subsection{Propriedades mecânicas dos painéis}

Os valores médios de ligação interna, módulo de elasticidade e módulo de ruptura em flexão estática estão apresentados no Quadro 4.

Com base nos resultados do Quadro 4, pode-se constatar que houve nítida tendência de aumento na ligação interna com o aumento no conteúdo de resina dos painéis. Observa-se também, nesse quadro, que os painéis produzidos com densidade de $0,80 \mathrm{~g} / \mathrm{cm}^{3}$ e $6 \%$ de resina apresentaram valor médio estatisticamente inferior em relação às demais condições de manufatura. Essa diferença pode ser atribuída à menor disponibilidade de resina em função do aumento da quantidade de partículas, além da menor quantidade de resina aplicada. Os demais tratamentos apresentaram valores médios de ligação interna superiores ao valor mínimo exigido pela norma CS 236-66 (1968) de $4,2 \mathrm{kgf} / \mathrm{cm}$. Outra constatação importante referese ao aumento na ligação interna para painéis com maior densidade. Esse resultado comprova a influência da maior compactação das partículas na qualidade da adesão entre as partículas.

Quanto ao módulo de elasticidade, embora não tenham sido constatadas diferenças estatísticas entre os tratamentos, pode-se verificar uma tendência de aumento dos valores médios dessa propriedade com

Quadro 4 - Valores médios das propriedades mecânicas dos painéis

Table 4-Average values of board mechanical properties

\begin{tabular}{lccc}
\hline Tratamento & $\begin{array}{c}\mathrm{LI} \\
\left(\mathrm{kgf} / \mathrm{cm}^{2}\right)\end{array}$ & $\begin{array}{c}\text { MOE } \\
\left(\mathrm{kgf} / \mathrm{cm}^{2}\right)\end{array}$ & $\begin{array}{c}\text { MOR } \\
\left(\mathrm{kgf} / \mathrm{cm}^{2}\right)\end{array}$ \\
\hline $\mathrm{T} 1(\mathrm{D}=0,6 / \mathrm{R}=6)$ & $4,69 \mathrm{~b}$ & $16.734 \mathrm{a}$ & $120 \mathrm{ab}$ \\
$\mathrm{T} 2(\mathrm{D}=0,6 / \mathrm{R}=8)$ & $7,21 \mathrm{bc}$ & $14.630 \mathrm{a}$ & $103 \mathrm{a}$ \\
$\mathrm{T} 3(\mathrm{D}=0,8 / \mathrm{R}=6)$ & $2,32 \mathrm{a}$ & $17.333 \mathrm{a}$ & $137 \mathrm{ab}$ \\
$\mathrm{T} 4(\mathrm{D}=0,8 / \mathrm{R}=8)$ & $5,31 \mathrm{~b}$ & $22.208 \mathrm{a}$ & $177 \mathrm{~b}$ \\
\hline
\end{tabular}

Médias seguidas pela mesma letra não diferem estatisticamente entre si, a $95 \%$ de probabilidade.

$\mathrm{D}=$ densidade nominal do painel $\left(\mathrm{g} / \mathrm{cm}^{3}\right)$ e $\mathrm{R}=$ conteúdo de resina $(\%)$. $\mathrm{LI}=$ ligação interna e $\mathrm{MOE}=$ módulo de elasticidade e $\mathrm{MOR}=$ módulo de ruptura. o incremento da densidade dos painéis e conteúdo de resina. Os painéis produzidos com densidade de $0,80 \mathrm{~g} / \mathrm{cm}^{3}$ e $8 \%$ de resina apresentaram valores médios superiores de MOE em comparação com os demais tratamentos.

Com relação ao módulo de ruptura, pode-se observar uma tendência de aumento dessa propriedade, com o incremento na densidade dos painéis produzidos com o mesmo conteúdo de resina. Entre os painéis produzidos com densidade de $0,80 \mathrm{~g} / \mathrm{cm}^{3}$, a aplicação de maior quantidade de resina resultou em valores superiores de MOR. Os painéis produzidos com densidade de $0,80 \mathrm{~g} / \mathrm{cm}^{3}$ e conteúdo de resina de $8 \%$ apresentaram maior resistência em flexão estática (MOR), em comparação com os painéis produzidos com menor densidade e conteúdo de resina. É importante ressaltar que, com exceção dos painéis produzidos com densidade de $0,80 \mathrm{~g} / \mathrm{cm}^{3}$ e conteúdo de resina de $6 \%$, os demais tratamentos apresentaram valores médios de MOR superiores em comparação com o valor mínimo de $112 \mathrm{kgf} / \mathrm{cm}^{2}$, exigido pela norma CS 236-66 para painéis aglomerados comerciais.

Os resultados de módulo de elasticidade e módulo de ruptura obtidos nesta pesquisa foram superiores em comparação com os resultados apresentados por Iwakiri et al. (2000) para painéis aglomerados de eucalipto com densidade de $0,70 \mathrm{~g} / \mathrm{cm}^{3}$.

\section{CONCLUSÕES}

Com base nos resultados obtidos nesta pesquisa, as seguintes conclusões podem ser apresentadas:

- A estabilidade dimensional dos painéis aglomerados de Grevillea não foi afetada significativamente pelos fatores densidade do painel e conteúdo de resina.

- Verificou-se um aumento na ligação interna para painéis produzidos com maior conteúdo de resina.

- Os painéis produzidos com densidade de 0,80 $\mathrm{g} / \mathrm{cm}^{3}$ e conteúdo de resina de $8 \%$ apresentaram melhores resultados de módulo de elasticidade e módulo de ruptura em flexão estática.

- Com exceção aos painéis produzidos com densidade de $0,80 \mathrm{~g} / \mathrm{cm}^{3}$ e conteúdo de resina de $6 \%$, todos os demais tratamentos apresentaram valores médios 
de resistência à flexão estática (MOR) superiores ao valor mínimo estabelecido pela norma CS 236-66 para painéis aglomerados comerciais.

- Os resultados indicaram que a madeira de Grevillea pode ser utilizada como fonte alternativa de matéria-prima para produção de painéis aglomerados com densidade de $0,80 \mathrm{~g} / \mathrm{cm}^{3}$, um pouco superior à densidade de painéis comerciais produzidos com densidade na faixa de 0,65 a $0,70 \mathrm{~g} / \mathrm{cm}^{3}$.

\section{AGRADECIMENTOS}

Os autores expressam seus agradecimentos à empresa BORDEN QUÍMICA IND. ECOM. LTDA. pela doação da resina uréia-formaldeído e à EMBRAPA - FLORESTAS pela disponibilização da madeira de Gravillea robusta utilizada nesta pesquisa.

\section{REFERÊNCIAS BIBLIOGRÁFICAS}

\section{AMERICAN SOCIETY FOR TESTING AND MATERIALS. ASTM. Standard methods of evaluating properties of wood-base fiber and particles materials. Philladelphia: 1982. (ASTM, 1037)}

COMMERCIAL STANDARD. Mat formed wood particleboard. CS 236-66. 1968.
IWAKIRI, S. et al. Mistura de espécies na produção de chapas de partículas estruturais "waferboard". Revista do Setor de Ciências Agrárias, v.14, n.1-2, p.107-114, 1995.

IWAKIRI, S. et al. Resíduos de serrarias na produção de painéis de madeira aglomerada de eucalipto. Scientia Agraria, v.1, n.1-2, p.23-28, 2000.

IWAKIRI, S. et al. Produção de chapas de madeira aglomerada de Pinus elliottii com inclusão laminar. Cerne, v.5, n.2, p.86-94, 1999.

KELLY, M.W. Critical literature review of relationships between processing parameters and physical properties of particleboard. Wisconsin: FPL, 1977. 66p. (U.S. Department of Agriculture).

MALONEY, T.M. Modern particleboard \& dry process fiberboard manufacturing. San Francisco: Miller Freeman, 1993. 681p.

MOSLEMI, A.A. Particleboard: Materials. Illinois: Southern Illinois University Press, 1974. v.1.244p.

TSOUMIS, G. Science and technology of wood. Structure, properties, utilization. New York: Chapman \& Hall, 1991. 494p. 